\title{
TESTING AND TARGETING A MOBILE APPLICATION FOR CREATIVES
}

by

\author{
Jack Hopkins \\ A Major Research Project
presented to Ryerson University
in partial fulfillment of the
requirements for the degree of
Master of Digital Media
in the Program of Digital Media
}

Bachelor of Journalism, Ryerson University, Toronto, Ontario, 2018

Toronto, Ontario, Canada, 2019

(C) Jack Hopkins, 2019 


\section{AUTHOR'S DECLARATION FOR ELECTRONIC SUBMISSION OF A MRP}

I hereby declare that I am the sole author of this MRP paper, within the Group MRP that includes Christian Bebis, for which he also has his own MRP paper. This is a true copy of my MRP paper, which fulfils my sole individual portion of our Group MRP, including any required final revisions.

I authorize Ryerson University to lend this MRP to other institutions or individuals for the purpose of scholarly research.

I further authorize Ryerson University to reproduce this MRP by photocopying or by other means, in total or in part, at the request of other institutions or individuals for the purpose of scholarly research.

I understand that my MRP may be made electronically available to the public 
"TESTING AND TARGETING A MOBILE APPLICATION FOR CREATIVES"

\author{
Jack Hopkins \\ Master of Digital Media \\ Digital Media \\ Ryerson University, 2019
}

\begin{abstract}
:
Will another social media application that aims to connect creators have a chance at success in our crowded digital landscape? We are putting that question to the test, by working on our idea of helping anyone find specific workspaces or spots that help them do their craft. Within a mobile application, letting users quickly find and post such spaces in their community while being able to connect with other users behind those posts, is what our ideas (and subsequent research) led us to. From target market and user experience interviews we have conducted for nearly a year, we believe that the need for such a mobile application among creatives justifies the work that must go into creating it.
\end{abstract}

lii. 


\section{Table of Contents}

Author's Declaration ii

Abstract iii

Table of Contents iv

List of Tables $\mathrm{V}$

List of Plates vi

Background of the Group MRP 1

Our expected joint product 2

- The unique value proposition

- Our Ideal Customer Persona

- Our competitive advantage and differentiating factors

- Our work so far

- What we need to reach our milestones

My contributions to this Group MRP 7

User Testing Research 7

Social Media Marketing 13

Legal Concerns 14

- Copyright

- Liability

User Pricing 16

Conclusion and Next Steps 16

Reference List 18

Glossary 21 


\section{List of Tables}

\section{Description}

Table 1 - Competitor Analysis

Table 2 - Our Key Performance Indicators

Table 3 - 1st Round Target Market Feedback

Table 4 - Prototype Version 2 iterations

\section{Page number}

$$
4-5
$$

6

$8-9$

$12-13$

V. 


\section{List of Plates}

Plate 1 - Moodboard information 10

Plate 2 - Prototype Versions 1 and 2 side-by-side 12

vi. 


\section{Background of the Group MRP}

StudioGo is a pre-revenue stage startup that was initiated in the Fall 2018 semester by several MDM students in a class together. The team members, which include Christian Bebis and me for this Group MRP, found a common interest in helping creative-minded professionals. After beginning target market research and ideation, the idea behind StudioGo evolved into: Solving a problem by creating a mobile application that provides peer-to-peer info on free, paid and publicly-accessible spaces for creative professionals who need a particular type of environment to work on their craft (\#1). From this hypothesis-- that a sufficient number of creative students (who are the target market) would find such an application useful-- the research on this potential customer segment began in October 2018 (\#2). As a result of furthering our research and feedback, along with identifying what each team member's strengths and interests are, the objective our team has fleshed out is: to build a solution that puts the power into the hands of users by offering a peer-to-peer location scouting community. Creatives need help finding adequate and affordable workspaces, while being able to inexpensively connect with their peers. Therefore, our proposed solution takes the form of an app that gives creative professionals or hobbyists an easy platform with information on any relevant space nearby, either posted by businesses or by private individuals, that the user can discover and then use to advance their craft. Through our UX processes, we have designed the app to provide feedback, ratings and an opportunity to connect with other users. In terms of relevance to Ryerson University's Master of Digital Media program: being able to strengthen this target market's opportunities to explore their professional and creative abilities is at the heart of our university's values and goals for its students. Every successful applicant to this program needs to have demonstrated enough of their own self-initiative and drive in their creative pursuits. Assisting other individuals in finding environments that are conducive to them achieving their own creative pursuits is highly relevant to the MDM program. 


\section{Our expected joint product}

Our Group MRP will have as its primary finished product a working Beta application. Its main purpose is as a platform for creative professionals or artists to find various types of spaces that they can use to work on their hobby or craft. Its locations can be added by other users, allowing greater interaction among users (and thus other professionals in the field) and the finding of ideal locations on a mass scale. It will only be on iOS, given the timescales of our Group MRP and the coding talent we have, which includes Matthew Bebis, who is the brother of Christian and a computer engineering student at Guelph University. Having as much target market data as possible to back up every design choice we have made is part of our final showcase of the Group MRP.

8. a) The unique value proposition: StudioGo's UVP has been developed by our founding team, our classmates during our presentations and other classwork, as well as from the feedback and data we have received during our first round of User Interviews, which is further explained in Section 10 of this paper. That proposition is: a location scouter that finds the spaces a user needs but did not know existed by crowdsourcing all the best spots near them. This will take the form of an app that we pitch as being "for creative professionals to share their creative insights in a peer-led community, with info on any nearby space that they can discover and use to advance their craft. We are your in-pocket location scout,"(\#1). A community that within the bounds of civility and good-faith collaboration, can share experiences and portfolios to form bonds, all with the same need for creative environments, as well as being a trustworthy way for venue owners to promote their space. The latter point is explained in further detail in Section 12 (b) below.

Our Ideal Customer Persona: Our primary target demographic is creative students/professionals, and our secondary one includes those interested in the 
short-term allocation of their property as a temporary creative workspace, which is a broader grouping. This was determined largely from our first round of User Interviews. As we initiated StudioGo during our studies, we chose creative students/professionals as our primary target market since we have easy access to them. That factor, along with the fact that our entire founding team is part of our target market, made it easier to identify with and understand what potential solutions they would gravitate to. As such, we created a Customer Persona template in the fall of 2018 that provided a basis for us to reach out to and gather research data from those we believed to be in our target market (\#3, slide 4). The findings are explored in further detail at the beginning of Section 10.

Our competitive advantage and differentiating factors: When analysing competitive advantage, we must look at the current solutions our target market uses to find places to complete their work, to gain exposure for their work, and to find others to collaborate with. These solutions include:

Social Media (\#4): Creatives currently use social media pages such as Facebook, Twitter and Instagram to discover new spaces to shoot and promote their own work.

Attending Events (\#4): Our customer segments attend various events to promote their work, learn about new opportunities, and find new and innovative ways to solve their problems, which include finding spaces, connecting with others \& promoting their work.

Our competition: Many of our potential users are not using solutions that fix their exact problem. From listening to the needs they have told us about in interviews, we are confident that we are developing a product best suited for them. Our competitive advantage is: peer-to-peer discovery of nearby spaces with rating systems, so users know they are not having places dishonestly promoted or pushed on them. By being creative students ourselves, we maintain an active presence in the field of our target 
market. This facilitates the design thinking process by allowing us to focus on customer needs, as we often share their problems. In terms of competing applications and services currently available, an example is Breather, which focuses on quantity of venues it selects. It does this by highlighting the long list of available workspaces that a user can select from, without featuring such variety as indoor vs. outdoor, free vs. paid, or business-posted vs. user-posted locations (\#5). A large part of this is due to StudioGo's Beta MVP having the key functionality of allowing anyone (within certain safeguards as described in Section 12.b) to post a location, regardless of them being part of a registered business. Here is an outline of three competitors we identified:

\section{Breather:}

Description: Modern workspaces you can keep for hours, days or months. Workspace on Demand.

Our Advantage: StudioGo will provide users with more variety. Breather only includes condos, homes, and residential areas. StudioGo plans to includes locations tailored to artist needs. From studio lofts, to recording studios, photo studios, and whichever other places our users value enough to add to our app.

Website: breather.com (\#6)

SetScouter:

Description: Find unique film locations faster.

Our Advantage: We expect StudioGo to provide more variety of locations in other industries, beyond only the need of providing photographers and videographers with locations.

Website: setscouter.com (\#7)

Ssshake:

Description: Ssshake is an international and inclusive cross-disciplinary creative network (\#8). 
Our Advantage: StudioGo's advantage will allow users to stay within one application to find the spaces they need, communicate with other creatives and post their work on an all-in-one platform, versus Ssshake's narrower focus on connecting creative professionals.

Website: ssshake.com (\#9).

Table 1 - Competitor Analysis

StudioGo's aim is to let users provide their own input, share experiences and connect to social media, all in order to create a community of artists who promote quality spaces. Since starting our competitor research last fall, we have not found an existing company that allows creative students and professionals to freely find a wide variety of locations to further their hobbies or crafts, which can be sourced by their peers in addition to businesses, thereby also increasing their ability to connect with like-minded creatives. As StudioGo's advantage over Ssshake demonstrates, centralizing all of these aspects under a coherent and easy-to-use design is what we believe will make the biggest impact in professional practice, and thereby have the most measurable effect on our target market as they pursue their creative dreams-- in whichever form those projects may take. When I began asking this very question for my portion of our third round of User Interviews, $100 \%$ of respondents agreed with that statement (\#10). Due to the flexibility \& variety of locations that we plan to showcase on the platform --which will be resourced from community peers -- along with the free aspect we hope to leverage through this wide variety of places that are posted (with lack of funds being a key concern for our target market, as Section 10 demonstrates), we feel that these factors give us a significant UVP over any competitors. Testing this hypothesis with more in-depth target market interviews beyond our Group MRP, and gauging this feedback alongside the launch of our Beta application will help us see if our research and work are truly breaking new ground in professional practice or yield a competitive product in the business landscape. 
Our work so far: The following table provides a summary of our work:

Our Key Performance Indicators (KPIs):

Round 1 Customer Interviews - Completed December 2018

First Adobe XD prototype - Completed March 2019

Round 2 Customer Interviews - Understanding the UI - Completed April 2019

Prototype Updates - Completed April 2019

Round 3 Customer Interviews - User Testing - Completed July 2019

Studiogo Newsletter /Creative Features campaign- June 2019- Ongoing

Working Beta - based on UI updates - Completed August 2019

Beta Updates - Fall 2019

Wide Launch (Beta) - Fall 2019

Incorporate - TBD (depending on the technical progress of our Beta MVP)

Table 2 - Our Key Performance Indicators

\section{What we need to reach our milestones}

We have determined that our most pressing needs to actualize our Beta MVP's readiness for a wide launch involve acquiring enough funding to:

A) obtain IT servers and a developer account, which would allow our app to process the amount of data that we hope we would get from launching it;

B) pay incorporation fees and open a business bank account;

C) paying for the necessary website hosting fees; and

D) designing and printing various branding and marketing materials and initiatives.

For the wide release of our Beta, a priority for us is contacting the owners of locations (where applicable) in our app to get further information from them and determine if they wish to create any promotional opportunities with us.

6. 


\section{My contributions to this Group MRP}

From the work Christian Bebis and I have succeeded in doing as part of StudioGo, we have both found different areas to work on for the Group MRP. I have a network of friends and acquaintances who fit the demographic of our target market. Excluding user interviews done by the rest of our team, I have completed 29 of such formal interviews over the multiple rounds of user discovery and user testing. As a result, a large part of the focus for my individual role within this Group MRP has been on gathering a sufficient amount of this feedback and data from these individuals (as well as finding more of them) by continuing these interviews as our Beta MVP progresses towards launch. Another one of my priorities is to conduct this data-gathering for potential locations, that may wish to be featured on our digital application and to use it, both in order to improve the UX from their perspective, as well as to increase the number of useful data points for our users for the Beta release. From all the data I obtain from this outreach, using that to inform our social media strategy (i.e. the frequency and types of posts we publish, the appearance of the contests and other interactive campaigns we launch, etc), as well as informing how we refine the visual branding we use in vital documents like our pitch deck, all become components of my individual role within the Group MRP.

\section{User Testing Research}

StudioGo has gone through three rounds of user testing research (\#11). The first round focused on gathering feedback from creative students during the Fall of 2018, when we were beginning to develop the idea behind StudioGo. From the script we made for the first round of interviews (\#12), the main findings were based around the following categories: "Pains", which considers the issues and/or painpoints the interview subjects reported encountering, "Solutions", which considers what habits or actions the subjects reported using currently to alleviate these Pains, and "Channels Used", which considers 
what specific tools, services, or platforms the subjects reported using to alleviate the Pains. This table below compiles the feedback from all 38 interviews the team conducted at that time (\#13). Titles in red denote high rates of agreement:

\begin{tabular}{|c|c|c|c|c|c|}
\hline Pains & $\begin{array}{l}\text { \# with } \\
\text { Pain }\end{array}$ & Solutions & $\begin{array}{l}\text { \# Using } \\
\text { Solution }\end{array}$ & $\begin{array}{c}\text { Channels } \\
\text { Used }\end{array}$ & $\begin{array}{l}\text { \# Using } \\
\text { Channels }\end{array}$ \\
\hline Money & 22 & $\begin{array}{c}\text { Attend local } \\
\text { events }\end{array}$ & 8 & Linkedln & 11 \\
\hline $\begin{array}{l}\text { Connections } \\
\text { (Industry/ } \\
\text { Creative) }\end{array}$ & 18 & $\begin{array}{l}\text { Branch out } \\
\text { to another } \\
\text { field }\end{array}$ & 5 & Indeed & 1 \\
\hline Workspaces & 15 & $\begin{array}{l}\text { Create own } \\
\text { workspaces }\end{array}$ & 11 & Email & 5 \\
\hline Education & 5 & $\begin{array}{c}\text { Further } \\
\text { education }\end{array}$ & 21 & $\begin{array}{c}\text { Social } \\
\text { Media } \\
\text { (Twitter, } \\
\text { Instagram, } \\
\text { Facebook) }\end{array}$ & 17 \\
\hline Mentorship & 5 & Self-market & 9 & Calling & 1 \\
\hline $\begin{array}{l}\text { Identifying } \\
\text { Other } \\
\text { Creatives }\end{array}$ & 2 & $\begin{array}{c}\text { Rent } \\
\text { monthly } \\
\text { studio } \\
\text { space }\end{array}$ & 3 & $\begin{array}{l}\text { Governme } \\
\text { nt funding }\end{array}$ & 7 \\
\hline
\end{tabular}

8. 


\begin{tabular}{|l|l|c|c|c|c|} 
Equipment & 2 & Internships & 4 & Other & 2 \\
\hline Jobs & 10 & Home & 10 & $/$ & $/$ \\
\hline $\begin{array}{l}\text { Portfolio } \\
\text { Building }\end{array}$ & 1 & Find a job & 5 & $/$ & $/$ \\
\hline $\begin{array}{l}\text { Location } \\
\text { (Transit or } \\
\text { Home) }\end{array}$ & 3 & $\begin{array}{c}\text { Use public } \\
\text { spaces }\end{array}$ & 12 & $/$ & $/$ \\
\hline Competition & 5 & $/$ & $/$ & $/$ & $/$ \\
\hline Self-Doubt & 1 & $/$ & $/$ & $/$ & $/$ \\
\hline Time & 2 & $/$ & $/$ & $/$ & \\
\hline
\end{tabular}

Table 3 -1st Round Target Market Feedback

The data are arranged with the most commonly reported results at the top in descending order. The most commonly reported Pains were, in order: -money/finances (22 of them, 58\%), -connecting in their industry (18 of them, 47\%), and -finding workspaces (15 of them, 39\%). The most commonly reported Solutions were, in order: -education (21 of them, 55\%), -using public spaces (12 of them, 32\%), and -using their home as a workspace (10 of them, $26 \%$ ). The most commonly reported Channels were, in order: -social media (17 of them, 45\%), -using Linkedln (11 of them, 29\%), and -accessing government funding (7 of them, 18\%).

Pivot: These results informed our team's pivot in November 2018 (\#14) toward a peer-to-peer resourced application that provides information on free and publiclyaccessible spaces to use, as opposed to being a platform that offers simply a 
marketplace of by-the-hour bookings for studio spaces and other short-term rentable locations. As well, removing the studio space-owners from this part of the customer research phase by pivoting in this way allowed us to remain focused on the customer segment we are most confident catering to. Upon solidifying StudioGo's idea and interest among our target market, the visual design and flow of our prototypes began to take shape. This includes StudioGo's Moodboard (\#15, slide 6), which is the overarching visual presentation of StudioGo's intended aesthetic, for our Beta MVP. It includes the following design choices:

\section{MoodBoard Information:}

Fonts: Proxima Nova Regular - Extra Bold, Paytone One Regular, Awesome Font (Brands, Logos, Regular)

Keywords: Happy, Energetic, Creative, Simple, Unique, Sleek, Adventurous, Modern Clean.

Colour Palette: A variety of warm colours included the hex codes: \#D2892A, \#EBC21A, \#A57182, \#FFF100, \#ED1C24, \#EE5B98.

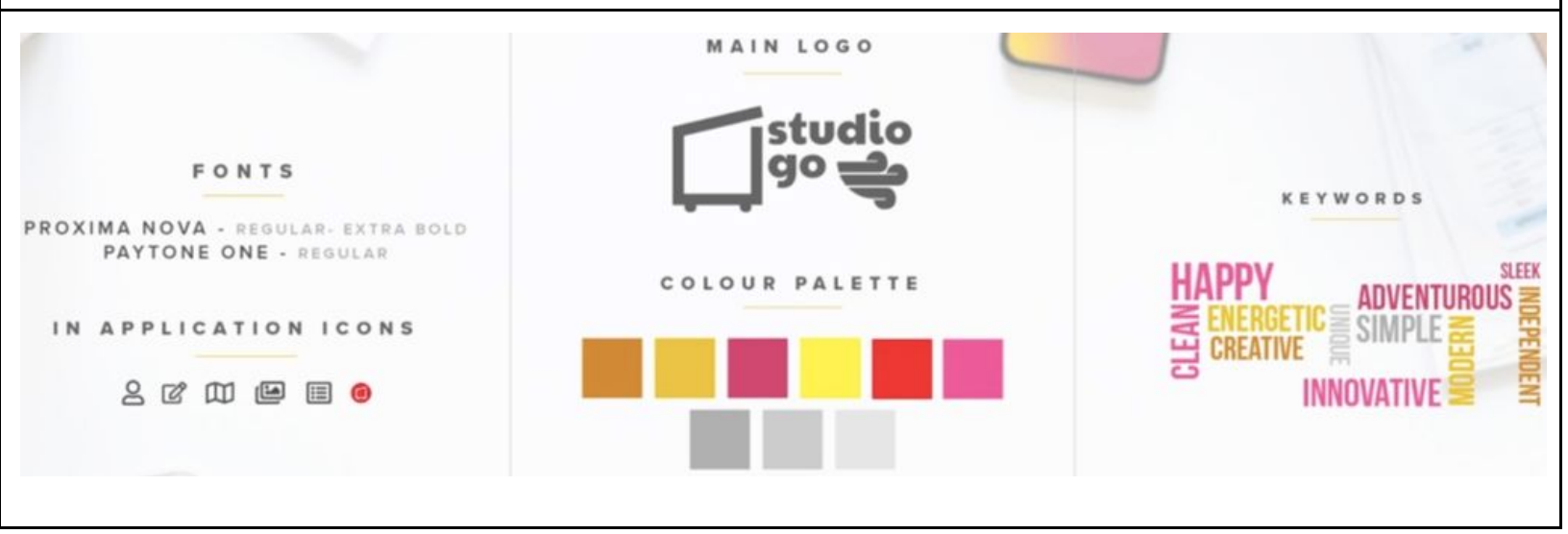

Plate 1 - Moodboard information

With the strong anecdotal feedback to these design choices in our moodboard that we received in our Introduction to Design Thinking class in the W2019 semester, we added that into our prototyping ideas, which were next to be tested. This led to our second round of our user testing research, this time focused not on the underlying idea of 10. 
StudioGo, but on the visual execution of it, via User Experience (UX) interviews. With our moodboards guiding StudioGo's first UX prototypes, consulting relevant academic material was important for us as well, including journals such as "Computers in Human Behavior", which includes the article "The negative impact of saturation on website trustworthiness and appeal" (\#16). When conducting UX interviews that involve the participants analysing and testing out a digital interface (in this case StudioGo's app prototypes), it is important to consider the ways in which they are likely to learn best. This article (\#16) by Alexander Skulmowski et al, considers how different amounts of colour and content saturation can affect users' ability to navigate and comprehend the information on various websites they are shown. Sixty-seven participants were shown 50 websites for varying lengths of time, up to ten seconds (\#16, p. 390).

"The post-hoc test for the interaction between saturation and content domain shows that a higher saturation...of content and lower...image density led to significantly lower trustworthiness and accessibility ratings...In short, we found negative effects of high saturation for usability depending on the content domain... Furthermore, we propose a temporal model of website perception based on the results in which users first evaluate the visual appeal of a website, followed by a continuous re-assessment of apparent usability, and its trustworthiness."

In creating a social platform that aims to accommodate these noted mental habits of the average user, we have taken such findings into account. For example, the script template I use for our UX interviews begins with direct questions about what pages they prefer most (with prompts such as "does it looks nicest, most useful?") (\#17), as well as what specific buttons, text, other visual features on certain pages they find confusing, or that they otherwise dislike. The first script template with our intended target market was made for the first round of user testing research in the entrepreneurship class, where the idea for StudioGo originated. When designing that prototype, we aimed to make it into the full template to act as the final guide for our Beta MVP. Using the Stanford Design Thinking Model (\#18) that we were introduced to in that class, we found that its template is most relevant for the target market outreach. Through reminders of how to "Empathize" and "Observe" when analysing your target market's problems and 
perspectives, it highlights the major points a designer should follow in the process of gathering (and then incorporating) user feedback into their product, in order to improve it.

During this process of February and March 2019, we interviewed 10 people in our target market (\#19). For the user interface, respondents liked all the major pages (the map page, location info page, profile page) as well as key components such as the search function (\#19, slide 3). Having a distinction between public and private locations was another feature that was approved of by all interviewees. The iconography we used for the buttons, as well as the placement of certain buttons and the visual distinction of the filters were confusing to a majority of respondents, and therefore these were among the main changes we made to StudioGo's second prototype (\#19, slide 4-5). The key changes are shown in the following side-by-side comparison:

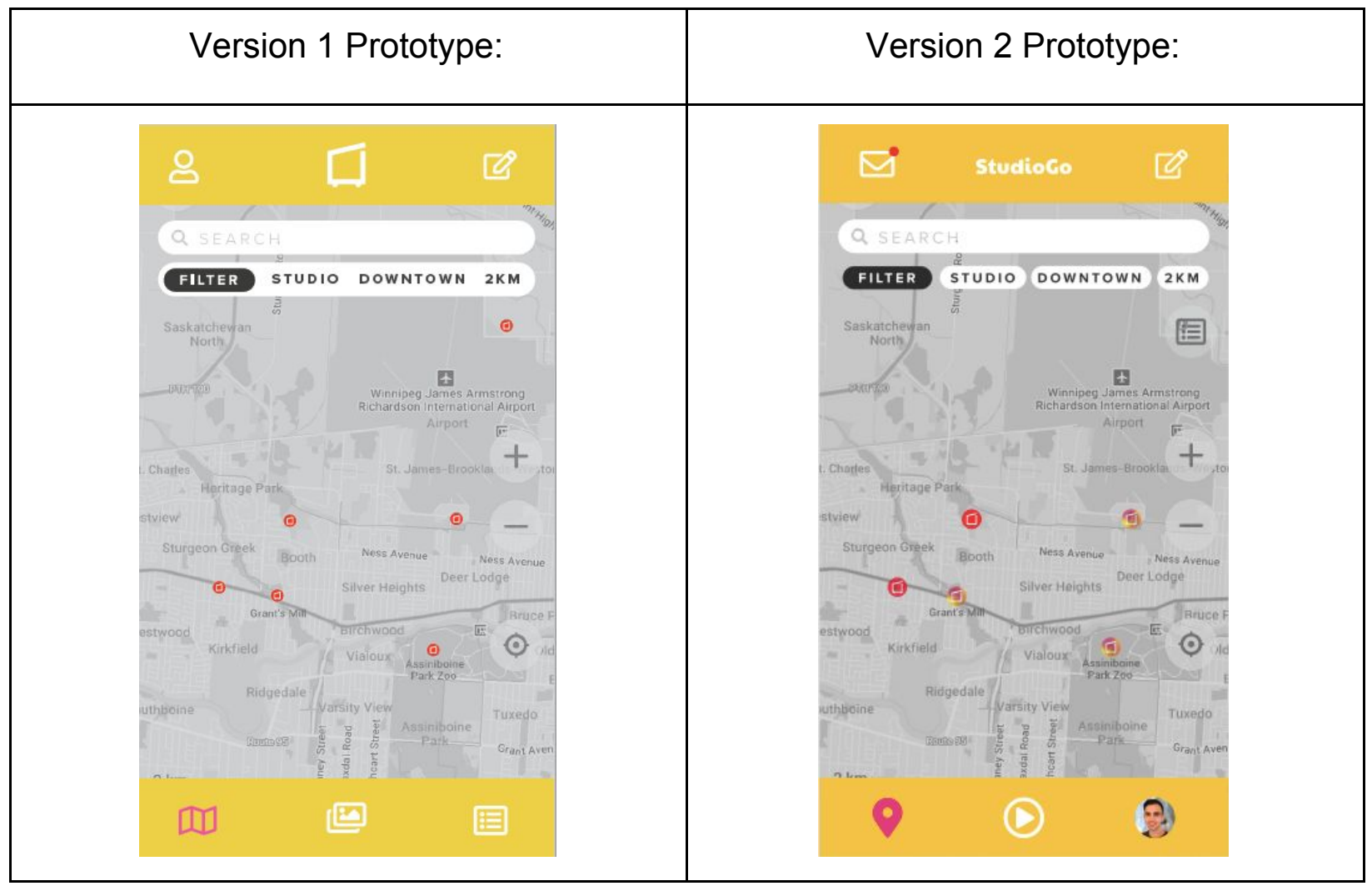

Plate 2- Prototype Versions 1 and 2 side-by-side 
Key Changes:

-added 'StudioGo' branding and profile photo to the navigation bars.

-separated the "Filter" bubble from the individual criteria (the white bubbles), to convey that they are additional filters, instead of simply 'switching' between them. -simpler/cleaner iconography along bottom bar.

Table 4- Prototype Version 2 iterations

\section{Social Media Marketing}

Upon securing the handle @thestudiogoapp on all StudioGo's social media platforms to ensure brand consistency, in June 2019 we initiated our personal artist social media campaigns, dubbed "Artist Features" (\#20). These are comprehensive portraits of the varied types of creative projects from relevant professionals in Toronto. The resulting social media postings consist of the creative's most prominent work as well as headshots and other identifying media (examples of which are not cited in this paper due to our Copyright obligations in Section 12.b). These are posted on StudioGo's Facebook and Instagram accounts, and the campaign overall has received interest from several creatives, whom we have subsequently profiled. As a result, the artist profiles we have included are no longer simply close friends of our founding team, but rather a diverse gallery of creators from across the city.

By promoting the creative work of Toronto's artists (without asking in any way for compensation from those profiled), and doing so while telling them of StudioGo's project, we plan to deepen the bonds StudioGo has with the local community. This will hopefully help us gain feedback more easily and widen the pool of 'launch ready' professionals in our target market, for when our application reaches the Beta MVP launch-ready stage. Having a sufficiently large number of active users will be the key factor in determining the success of our launch. Also, harnessing the knowledge of this 
group to find more StudioGo verified locations, as well as to generate more social media material are other goals for these Artist Features. From existing connections via creative groups such as Artery Toronto, which is a platform that sets up creative-focused events in eclectic venues across Toronto (\#21), we are confident we will be able to onboard the critical mass of users and locations to make the Beta MVP competitive.

\section{Legal Concerns}

There are certain legal questions concerning liability and intellectual property that we as StudioGo's founding team must clarify. Seeking relevant legal advice in the near-future for the following points is a priority.

Copyright: For the scope of our MRP, we clarified StudioGo's rights and obligations regarding when we accept social media content from others, and what intellectual property limitations we face. This pertains to our ongoing Artists Features campaign. When we initiated it, we did so with the understanding that all content we obtain from non-StudioGo parties to use remains within their control. Should they object or request changes to how their content is used, we agree to their wishes. Hypothetically, if we were unable to agree to their request, we would return all of their materials and ensure we do not use any of it, or keep any of it on our accounts, if it had already been posted.

Liability: The security concerns \& liabilities we face when accepting location suggestions is an issue that falls mainly outside our Group MRP work. However, answering the following point is still necessary before our app is launched widely. Verifying all location pins on the app to ensure user safety and trust is vital to the security and success of StudioGo. As our Beta MVP nears completion, we will more widely ask our target market (potentially via a social media campaign) to share their locations in Toronto that they have found helpful for working on their craft. We already 
have done this in our User Testing and UX interviews, although the mass scale of location options that we expect our widely-launched app may get suggests that we need to be able to narrow down these results to the best quality locations. Having a system where our team puts a 'hold' on all incoming location suggestions, for the necessary amount of time to verify and sort through them is a part of this. This will have the other benefit of assuring location owners that they can add their spot to a trustworthy platform. As we have at least several locations of sufficient quality (\#22), we will have a 'first wave' of locations ready to be added to our Beta MVP, regardless of how long a 'hold and check' has to be implemented by us. The following point is not within the scope of our Group MRP: knowing what our responsibilities are (as the founding team of StudioGo) for what liability we may face in situations where users have their safety compromised in some way at a location that we helped facilitate for them (e.g., they become injured at the location, they become victim to a crime there, etc). We will seek the relevant legal advice when a plan for the wide-launch of the Beta is formed, likely by contacting the RSU's legal team for direction. Another point not within the scope of our Group MRP involves incorporating, and determining at what point in time it becomes worth it to do so. When one or more entrepreneurs jointly incorporate a business, each of them is absolved of personal financial liability, meaning that the finances in their personal bank accounts will remain untouched in the event of them facing liability for any of their actions related to their corporation. This does not preclude them of any liability, as part of the incorporation process involves creating a business bank account for the corporation, which can face legal penalties in such an event. However, this important distinction between liability of personal assets and finances, versus liability of assets owned by one's corporation, is a strong incentive to pursue an incorporation. We are confident that upon our Beta MVP being launched and therefore being able to attract users \& potential business partners, incorporating is likely to be beneficial, and will therefore ensue. Of course, any research that demonstrates the attractiveness of StudioGo's value offering can only be validated by further actions of interest from our 15. 
target market, most importantly by them: A) using the app, and B) fulfilling the assumptions of our revenue model. Gathering such data will decisively answer the question of this MRP, which is our primary goal for the Beta launch.

\section{User Pricing}

Another important factor in the success of user uptake is choosing the ideal pricing model. Before implementing a transactional component, we want our users to feel valued and comfortable by considering StudioGo a helpful tool, rather than as only a path to consumption. The overwhelming preference from our interviews (\#10) has been to pursue a freemium model, which is a commonplace pricing strategy that offers a basic tier free of charge to users, with additional features offered at a fixed price. Based on that feedback, we intend the pricing strategy to be as follows:

We provide users free access to StudioGo, with additional features that allow them to improve their experience on the app for a price. This is known as the "Premium Membership", and it will be offered at $\$ 4.99$ per month. Our free tier will contain ads and include limited features. Users will be able to search for locations, add portfolio pieces \& connect with other creatives. To summarize the Premium tier's goal: by having various rewards systems in our revenue model, we believe we will grow a consistent user base. Part of that includes offering two free months of premium access, provided the new user includes credit card information, with the hope that this will entice them to try Premium Membership (and the app overall) for longer. More details on features in the Premium tier can be found on slide 6 of our Pitch Deck (\#23).

\section{Conclusion and Next Steps}

From everything discussed so far, our founding team (both for our Group MRP and for StudioGo as a startup) is confident that we can launch the Beta MVP version of our application before the end of the year. This requires continued development on the coding and IT infrastructure, which Bebis' brother, Matthew Bebis, is helping with. We 
have over two dozen locations on our master list of spots that we expect to be of sufficient quality to include on the initial Beta MVP (\#24), to ensure it is populated with information as soon as users begin to sign up during the launch phase. Hopefully, these factors will encourage users to add more of their own known locations and other content to the platform. Fulfilling these last two steps is key to realising any of the traction and impact that we are confident StudioGo can have, based on the wide variety of feedback we have gotten during our rounds of user interviews and from elsewhere. 


\section{Reference List:}

\#1. Bebis, Christian \& Hopkins, Jack. (2018). Step 19: Value Proposition. 100 Steps 2 Startup. Retrieved from https://drive.google.com/file/d/1WCvTKP-ruTY8xWTrL0hAf27W9 UML1AL/view

\#2. Bebis, Christian \& Hopkins, Jack. (2018). Entrepreneurship Update 2. Google Drive. Retrieved from https://docs.google.com/presentation/d/1XnKLht5ODSJeX1DelwXsC3ed-XufBufWFWZ M_4olsfk/edit?usp=sharing

\#3. Bebis, Christian \& Hopkins, Jack. (February 6, 2019). User Persona Presentation. Google Drive. Retrieved from https://drive.google.com/file/d/OB9FGUhqZUs9iQkZDQWZfTWILczhmdGZrSE9NOGFfZ khFSmVF/view?usp=sharing

\#4. Bebis, Christian \& Hopkins, Jack. (2018). Round 1 Interview Findings. Google Drive. Retrieved from https://docs.google.com/spreadsheets/d/195J3e8kF3k29zXDGYSNm6JTTFJuJaYMAjm s3KbVGTgA/edit?usp=sharing

\#5. (n.d.). Workspaces in Toronto. Breather. Retrieved from https://breather.com/locations/toronto

\#6. (n.d.). Retrieved from Breather.com

\#7. (n.d.). Retrieved from setscouter.com

\#8. (n.d.) About. Ssshake. Retrieved from https://ssshake.com/aboutssshake \#9. (n.d.). Retrieved from https://ssshake.com/

\#10. Bebis, Christian \& Hopkins, Jack. (2019). 2nd \& 3rd Round interviewee answers. Google Drive. Retrieved from https://docs.google.com/document/d/1c4hYPeCuAuFdep6jvCJpGnVnN2zgxTJsDeUY7 3FcCU8/edit?usp=sharing

\#11. Bebis, Christian \& Hopkins, Jack. (2019). User Interviews. Google Drive. Retrieved from 
https://drive.google.com/drive/folders/17TrSAOLicoVSIV988A9nOYfkPfrdPoQH?usp=sh aring

\#12. Bebis, Christian \& Hopkins, Jack. (2019). Pre-Prototype Interview Script. Google Drive. Retrieved from

https://docs.google.com/document/d/1TC60_4dgfbT1qPFiVZsuRK9ZCa6EwXxeQ3iDmTJONc/edit?usp=sharing

\#13. Bebis, Christian \& Hopkins, Jack. (2019). 1st Round Target Market Interview Chart (Anonymized). Google Drive. Retrieved from https://docs.google.com/document/d/14CtbfY7OmltlBa03rCU2r1qUQJZ9qRN9cbEpl4pv 9o0/edit?usp=sharing

\#14. Bebis, Christian \& Hopkins, Jack. (November 12, 2018). Update \#4 StudioGo. Google Drive. Retrieved from https://docs.google.com/presentation/d/1S2WL3fb0OLS6mu38G2pyFajp1fJkRQZrSoiC $\underline{\mathrm{cQhXzuA} / \mathrm{edit} \text { ?usp=sharing }}$

\#15. Bebis, Christian \& Hopkins, Jack. (2019). UX Final Presentation. Google Drive. Retrieved from https://docs.google.com/presentation/d/13KUkeeSkCVD28BK8Jfy8cU5HQktg6YjKkt6hn cnUf-I/edit?usp=sharing

\#16. Skulmowski, A., Augustin, Y., Pradel, S., Nebel, S., Schneider, S., \& Rey, G. D. (2016). The negative impact of saturation on website trustworthiness and appeal: A temporal model of aesthetic website perception. Computers in Human Behavior, 61(Complete), 386-393. doi:10.1016/j.chb.2016.03.054

https://journals-scholarsportal-info.ezproxy.lib.ryerson.ca/details/07475632/v61icomplet e/386 tniosotmoawp.xml

\#17. Bebis, Christian \& Hopkins, Jack. (2019). UX Interview Script. (2019). Google Drive. Retrieved from 
https://docs.google.com/document/d/1vVumc6pfD9TP8NUrt4MGN3M1apNvTKj-8isHE6 3RS30/edit?usp=sharing

\#18. Plattner, Hasso. (n.d.). An Introduction to Design Thinking Process Guide. Institute of Design at Stanford. Retrieved from

https://dschool-old.stanford.edu/sandbox/groups/designresources/wiki/36873/attachmen ts/74b3d/ModeGuideBOOTCAMP2010L.pdf

\#19. Bebis, Christian \& Cohen, Jonas \& Hopkins, Jack. UX March 27th 2018. Google Drive. Retrieved from

https://docs.google.com/presentation/d/1lhDzWAo22JZAck59hb-M8jpXK_zudBebc-gNa zrA6 0/edit\#slide=id.p

\#20. Bebis, Christian \& Hopkins, Jack. (June 3, 2019). StudioGo Presents Artist Features. Instagram. Retrieved from https://www.instagram.com/p/ByQTNGbhkPc/

\#21. (n.d.). Artery. Facebook. Retrieved from

https://www.facebook.com/Artery-682621958504214/about/?ref=page_internal

\#22. Bebis, Christian \& Hopkins, Jack. (2019). FINAL Locations for Beta. Google Drive. Retrieved from https://docs.google.com/document/d/1_yfeB0xT_Hv0JVAcVOt7R5s-x-F3rzbEfK2iRokul $1 \mathrm{Q} / \mathrm{edit}$ ?usp=sharing

\#23. Bebis, Christian \& Hopkins, Jack. (2019). StudioGo Pitch Deck. Google Drive. Retrieved from https://docs.google.com/presentation/d/1Ch1n9VzxkCahyYxNWqyHEWQzHVchO5OiB wfjazk26y0/edit?usp=sharing

\#24. Bebis, Christian \& Hopkins, Jack. (2019). All locations for Beta. Google Drive. Retrieved from https://docs.google.com/document/d/1K57KS2Uu6PIVXnOR-v3HonPCK7AMQ8GCuNU Ue1SX82c/edit?usp=sharing

20. 


\section{Glossary:}

"App" - a mobile software application.

"Beta MVP" - 'Beta Minimum Viable Product', ie. the first functioning version of our mobile application, as opposed to a non-functioning prototype, that merely gives the impression of how the application functions.

"Group MRP" - The Group Major Research Project that Christian Bebis and Jack Hopkins are completing for their Master of Digital Media degrees at Ryerson University, with this paper and the August 20 Showcase serving as the culminating deliverables for Hopkins, while Bebis completes his own paper with his own focus.

"MDM" - Ryerson's Master of Digital Media degree, of which Bebis and Hopkins are both currently enrolled.

"UVP" - Unique Value Proposition.

"UX"- User Experience.

"We" - The collective Group MRP team working the mobile application called 'StudioGo' as part of a startup, consisting of Christian Bebis and Jack Hopkins. 